\title{
Häufige infektiöse Enteritiden - Evaluationsbogen (Seite 1)
}

\section{A. Angaben zur Person: Bitte ausfüllen bzw. ankreuzen!}

Name, Vorname, akad. Titel:

Straße, Hausnr.: PLZ/Ort:

Ich bin Mitglied der Ärztekammer (bitte Namen der Kammer eintragen):

Jahr meiner Approbation (bitte eintragen):

Ich befinde mich in der Weiterbildung zum (bitte eintragen):

Ich habe eine abgeschlossene Weiterbildung in/für (bitte Fach eintragen):

Ich bin tätig als: $\square$ Assistenzarzt, $\square$ Oberarzt $\square$ Chefarzt $\square$ niedergelassener Arzt $\square$ Sonstiges (bitte eintragen):

Ich bin DMW Abonnent: $\square$ ja $\square$ nein

Falls nein: Ich habe den Fragebogen aus/von $\square$ Thieme-connect $\square$ Kollegen $\square$ der Klinik $\square$ einer Bibliothek $\square$ Sonstiges

\section{B. Teilnahmebedingungen}

Für diese Fortbildungseinheit erhalten Sie einen Fortbildungspunkt im Rahmen des freiwilligen Fortbildungszertifikates. Hierfür

- müssen 8 der 11 Fragen richtig beantwortet sein (bestanden hat auch, wer nicht weniger Fragen richtig beantwortet hat als der Durchschnitt aller Teilnehmer).

- müssen Seite 1 und Seite 2 des Evaluationsbogens vollständig ausgefüllt sein. Unvollständig ausgefüllte Bögen können nicht berücksichtigt werden!

- muss eine DMW CME-Wertmarke* im Feld E aufgeklebt sein.

\section{Bitte senden Sie}

- den vollständig ausgefüllten Evaluationsbogen (Seite 1 und 2) mit der aufgeklebten DMW CME-Wertmarke*;

- eine an Sie selbst adressierte und ausreichend frankierte Eingangsbestätigungskarte*;

- einen an Sie selbst adressierten und ausreichend frankierten Rückumschlag

an : Georg Thieme Verlag, DMW

Stichwort "CME"

Postfach 301120

70451 Stuttgart

Einsendeschluss ist der 04.07.2001 (Datum des Poststempels). Die Bestehensgrenze wird dann ermittelt. Die Zertifikate werden ab dem 11.07.2001 auf dem Postweg versandt. Von telefonischen Anfragen bitten wir abzusehen.

\section{Datenschutz}

Ihre Daten werden ausschließlich für die Bearbeitung dieser Fortbildungseinheit verwendet. Es erfolgt keine Speicherung der Ergebnisse über die für die Bearbeitung der Fortbildungseinheit notwendige Zeit hinaus. Die Daten werden nach Versand der Testate anonymisiert. Namens- und Adressangaben dienen nur dem Versand der Testate. Die Angaben zur Person dienen nur statistischen Zwecken und werden von den Adressangaben anonymisiert verarbeitet.

* gilt nicht für DMW Abonnenten, wir benötigen lediglich Ihre Abonnement-Nr. (bitte in das Feld E eintragen), siehe auch S. A 410

DMW Deutsche Medizinische Wochenschrift 126 (2001), 537

(c) Georg Thieme Verlag Stuttgart · New York

\section{Wichtige Hinweise}

- Die CME-Beiträge der DMW wurden durch die Nordrheinische Akademie für ärztliche Fort- und Weiterbildung anerkannt. Die DMW ist somit zur Vergabe der Fortbildungspunkte für diese Fortbildungseinheit im Rahmen des freiwilligen Fortbildungszertifikates berechtigt.

- Diese Fortbildungspunkte der Nordrheinischen Akademie für ärztliche Fort- und Weiterbildung werden von den anderen zertifizierenden Ärztekammern anerkannt.

- Die Vergabe der Fortbildungspunkte ist nicht an ein Abonnement gekoppelt!

\section{E.}

Bitte in dieses Feld die DMW CME-Wertmarke kleben

(Informationen zu den CME-Wertmarken S. S. A 410)

oder Ihre DMW Abonnement-Nummer eintragen:

(siehe Adressaufkleber Ihrer DMV)

\section{F. Zertifizierungsfeld* *}

\section{Ihr Ergebnis}

Sie haben von Fragen richtig beantwortet. Im Durchschnitt wurden ___ der Fragen richtig beantwortet. Sie haben $\square$ bestanden $\square$ nicht bestanden.

Stuttgart, den

Datum Stempel/Unterschrift

${ }^{* *}$ wird durch die DMW ausgefüllt 


\section{Häufige infektiöse Enteritiden - Evaluationsbogen (Seite 2)}

\section{G. Fragen zur Zertifizierung}

\section{a) Didaktisch-methodische Evaluation: Bitte ausfüllen!}

1 Die häufigen infelktiösen Enteritiden kommen in meiner ärztlichen Tätigkeit

$\square$ häufig vor

$\square$ regelmäßig vor

$\square$ selten vor

$\square$ gar nicht vor

2 Bezüglich der Diagnostik und Therapie der häufigen infektiösen Enteritiden gab es für mich vor dem Studium dieses Beitrages

$\square$ eine feste Gesamtstrategie

$\square$ noch offene Einzelprobleme (bitte benennen):

\section{$\square$ keine Strategie}

3 Bezüglich der häufigen infektiösen Enteritiden

$\square$ fühle ich mich nach dem Studium des Beitrages in meiner Strategie bestätigt

$\square$ habe ich meine Strategie verändert (bitte benennen):

$\square$ habe ich erstmals eine einheitliche Strategie erarbeitet

$\square$ habe ich keine einheitliche Strategie ableiten können

4 Wurden aus der Sicht Ihrer täglichen Praxis heraus wichtige Aspekte des Themas

a) nicht erwähnt? $\square$ ja $\square$ nein

Wenn ja, welche (bitte benennen)?

b) zu knapp abgehandelt? $\square$ ja $\square$ nein

Wenn ja, welche (bitte benennen)?

c) überbewertet? $\square$ ja $\square$ nein?

Wenn ja, welche (bitte benennen)?

5 Verständlichkeit des Beitrages

$\square$ Der Beitrag ist nur für Spezialisten verständlich

口 Der Beitrag ist auch für Nicht-Spezialisten verständlich

Deutsche Medizinische Wochenschrift 126 (2001), 538

(c) Georg Thieme Verlag Stuttgart · New York
6 Beantwortung der Fragen

$\square$ Die Fragen lassen sich aus dem Studium des Beitrages allein beantworten

$\square$ Die Fragen lassen sich nur unter Zuhilfenahme zusätzlicher Literatur beantworten

7 Die Aussagen des Beitrages benötigen eine ausführlichere Darstellung

¿ zusätzlicher Daten

$\square \quad$ von Befunden bildgebender Verfahren

$\square$ die Darstellung ist ausreichend

\section{b) Lernerfolgskontrolle: Bitte ausfüllen!}

Antworten zum Quiz (nur eine Antwort pro Frage ankreuzen)

\begin{tabular}{|l|l|l|l|l|l|}
\hline $\begin{array}{c}\text { Antwort } \\
\text { Frage }\end{array}$ & B & B & C & D & E \\
\hline 1 & & & & & \\
\hline 2 & & & & & \\
\hline 3 & & & & & \\
\hline 4 & & & & & \\
\hline 5 & & & & & \\
\hline 6 & & & & & \\
\hline 7 & & & & & \\
\hline 8 & & & & & \\
\hline 9 & & & & & \\
\hline 10 & & & & & \\
\hline 11 & & & & & \\
\hline
\end{tabular}

Die richtigen Antworten werden in der DMW Nr. 27/2001 (erscheint am 06.07.2001) veröffentlicht.

\section{H. Erklärung: Bitte ausfüllen!}

\section{Erklärung}

Ich versichere, dass ich die Beantwortung der Fragen selbst und ohne fremde Hilfe durchgeführt habe

Ort/Datum:

Unterschrift: 